High-Performance Computing

\title{
TEMPLET: A MARKUP LANGUAGE FOR CONCURRENT ACTOR-ORIENTED PROGRAMMING
}

\author{
S.V. Vostokin \\ Samara National Research University, Samara, Russia
}

\begin{abstract}
The article presents a markup domain-specific language (DSL) for concurrent programming. Runtime libraries and language extensions are the usual ways to implement parallel execution. However, their using often requires a special programming skill. The modern languages with build-in parallel constructs are more convenient for programming, but they are poorly integrated with existing high performance computing infrastructure. We propose a compromise solution which uses DSL together with $\mathrm{C}++$ programming language. The article discusses syntax, programming model, and some practical applications of the language.
\end{abstract}

Keywords: domain-specific language, parallel programming, actor model, language-oriented programming, skeleton programming.

Citation: Vostokin SV. Templet: a markup language for concurrent actororiented programming. CEUR Workshop Proceedings, 2016; 1638: 460-468. DOI: $10.18287 / 1613-0073-2016-1638-460-468$

\section{Introduction}

The Templet language is a domain-specific language (DSL). It is designed to be used together with a sequential procedural or an object-oriented programming language. The new property of the language is an implicit specification of the actor's computation semantics with a marked serial code. The language detailed description first appeared as arXiv preprint [1].

The article focuses on a design of the markup language. The design of the language basically follows the concept of the language-oriented programming [2,3]. The algebraic-like notation similar to the CSP formalism was applied to describe parallel processes and interactions [4]. The idea of a minimalistic design with emphasis on the basic abstractions is taken from the programming language Oberon [5].

The language design is based on the three concepts. The first one is so called active markup. Usually a markup is read form the source file and produces some effects in the target file (e.g. adding synchronization and/or communication commands). In our approach source and target is the same file. The language preprocessor overwrites 
files content. The markup inside the files directs the conversion to keep a desired code structure.

The second one is a programming model. We introduce a diffusive (with no locking) programming model that describes concurrent activity as a message exchange between parallel processes. They are activated by incoming messages. The channels define message exchange protocols. The model avoids concurrent data access, hence it is easier to use when multithreading. The model is a specialization of the actor formalism [6].

The third concept is based on description of concurrent activity with sequential code. This method is derived from formal theories that consider parallel process as set of behaviors (sequences of system states and/or atomic actions). We simulate the sequences with random number generator.

The following paragraphs illustrate these concepts with syntax and code examples. The article ends with an overview of related works. The experimental preprocessor for the Templet language and code samples in marked $\mathrm{C}++$ are available at http://github.com/templet-language.

\section{$1 \quad$ Active markup}

To describe the syntax, an extended Backus-Naur Formalism called EBNF is used. The following EBNF rules describe the block structure of a module. The module here is a unit of code that can include one or several files.

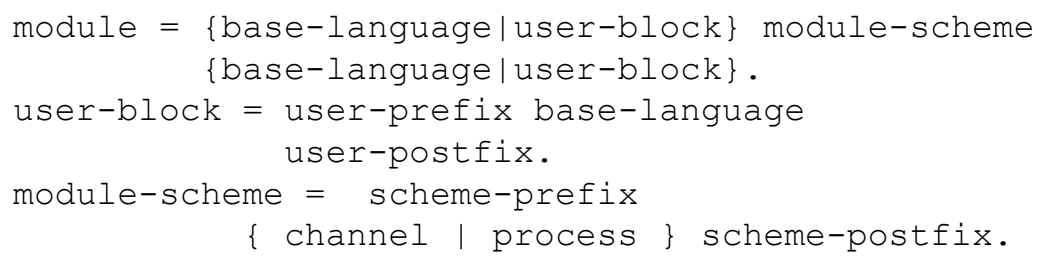

The code of a module consists of the single module scheme section and multiple code sections in $\mathrm{C}++$ language with highlighted user blocks. These sections are distinguished from the rest of the code by means of $\mathrm{C}++$ comments. For example, the marked C++ code may look as follows. The blocks' names according to the markup language syntax are shown on the right side.

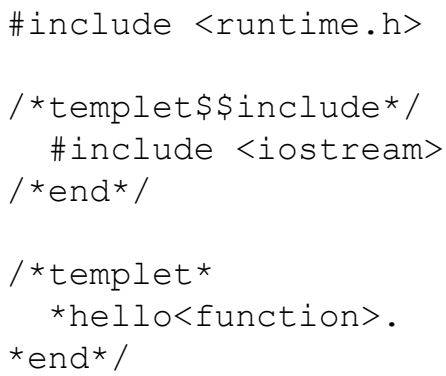




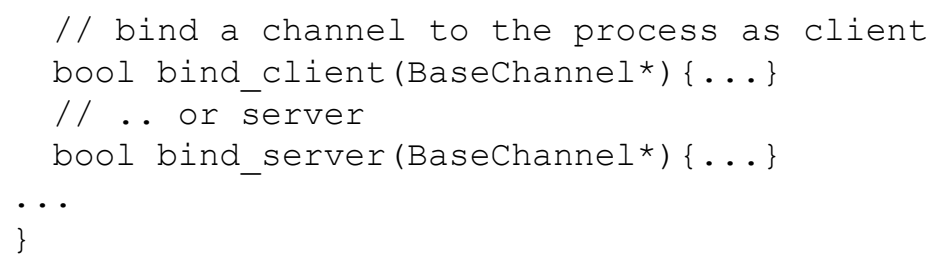

The BaseChannel has the following behavior. The access to the channel alternately belongs to pair of processes called client and server. The client process has access right to the channel in the beginning of computations. The methods access_client() and access_server() allow client or server to check for access. The methods send_client() and send_server() can be used to grant access from client to server or from server to client respectively.

The BaseProcess has the following behavior. The methods bind_client() and bind_server() establish connection between a process (as a client or as a server) and a channel. The method $\operatorname{recv}()$ is called at the moment getting access to the channel. The channel is passed as recv() argument.

The implementation also carries out the rules below. If the process gets access to multiple channels, it takes several consecutive calls to $\operatorname{recv}()$ in random order. If some process sends the channel access to another process, the other process will sooner or later get the access.

\section{Concurrent execution semantics}

The program implementation in $\mathrm{C}++$ language should provide the opportunity for a non-deterministic performance. The non-determinism of program execution is simulated by means of pseudo-random numbers.

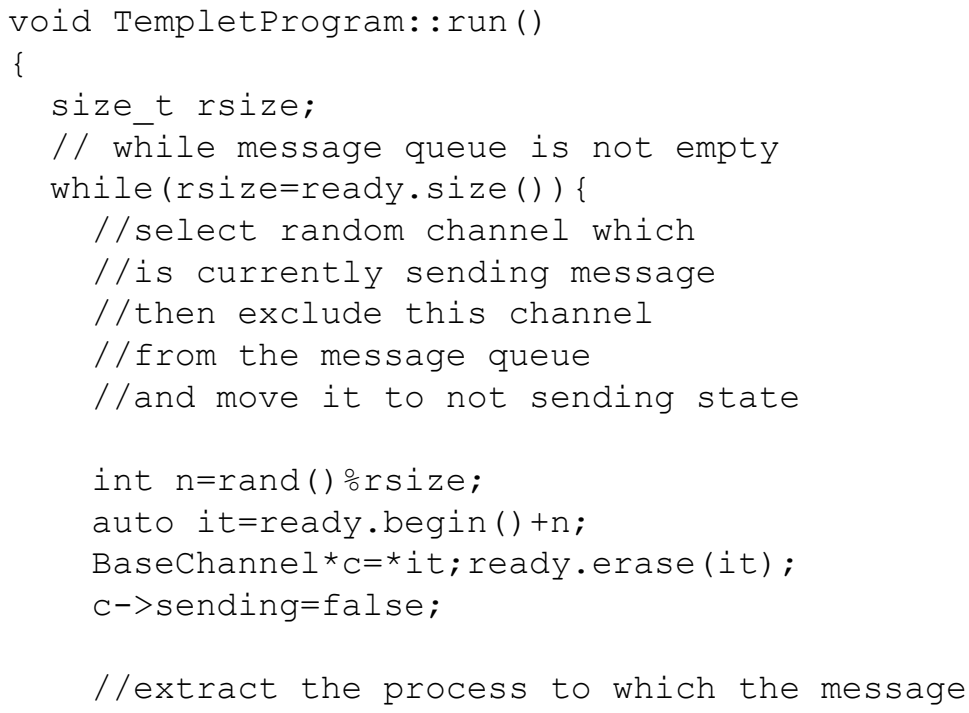




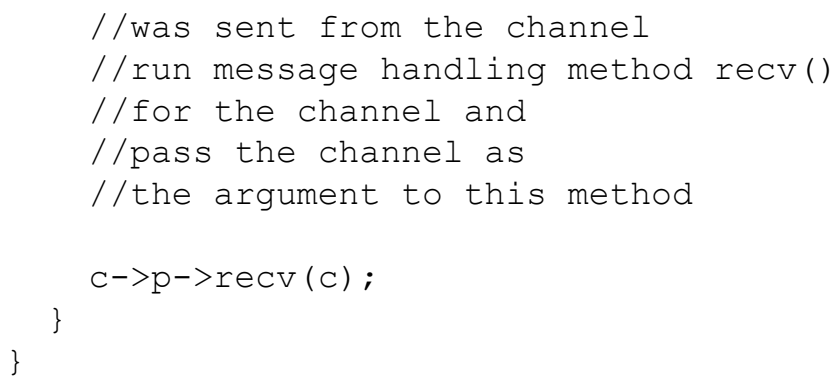

Appropriate runtime libraries are provided for truly parallel execution of a code. Some modifications to mapping algorithm may also be required.

\section{Module scheme syntax}

This is a complete EBNF description of module scheme in the Templet language.

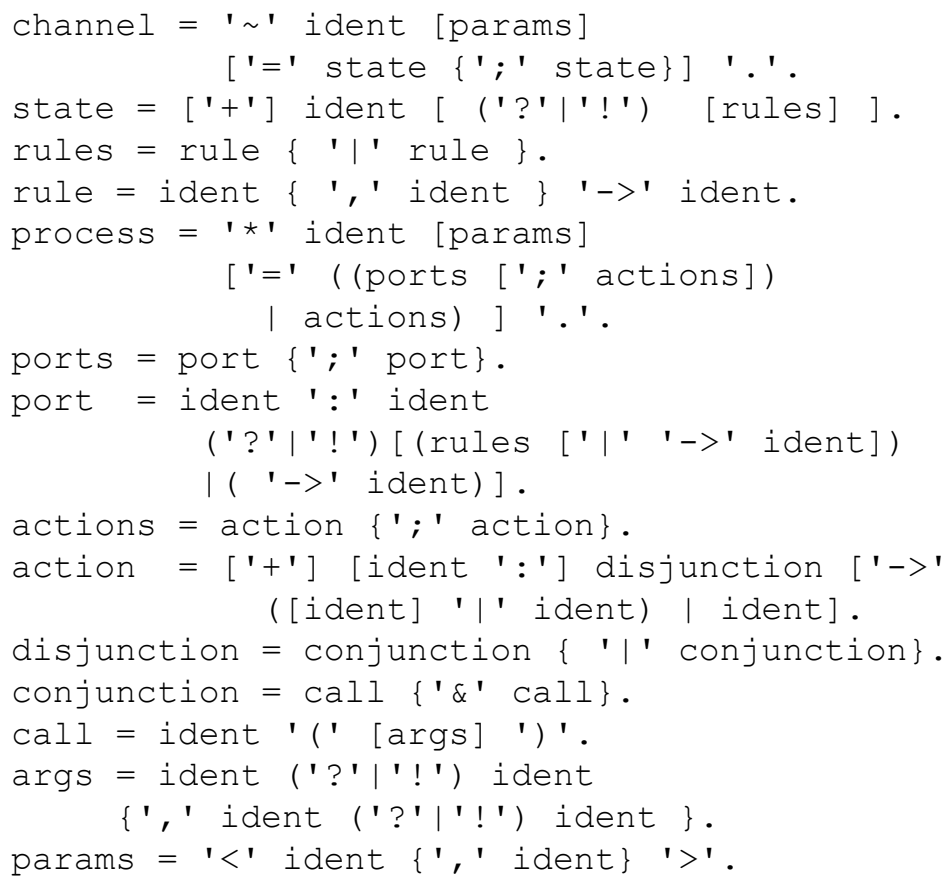

For example, there is a program that checks the trigonometric identity $\sin 2 x+\cos 2 x=1$ in parallel. The process of Master class sends $x$ values to working processes of Worker class via channels of Link class. The Master gets the squares of trigonometric functions and calculates their sum in return. The protocol Link to verify the trigonometric identity may be coded in Templet DSL like shown below.

$\sim \mathrm{Link}=+\mathrm{BEGIN}$ ? ArgCos $->\operatorname{CALCCOS}$ । 


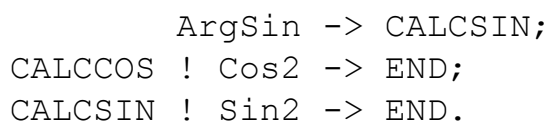

The Master and Worker processes for checking the trigonometric identity can be defined as follows.

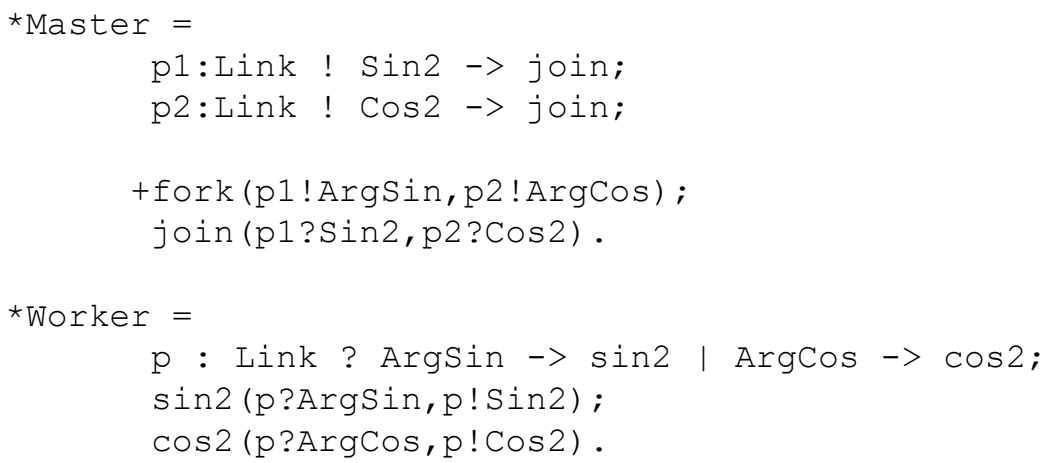

Any Templet program is a network of objects. Objects are instances of $\mathrm{C}++$ classes generated from the DSL. These C++ classes are in turn derived from BaseChannel class or BaseProcess class. The network of objects is coded manually in $\mathrm{C}++$.

\section{Applications overview}

The current implementation includes another three samples to illustrate the practical use of the Templet language for various applications.

The Gauss-Seidel method for solving the Laplace equation is the first one. This example illustrates the use of the language in the field of high performance scientific computing. It also shows how the Templet simulation runtime can help to predict program performance without an explicit mathematical model or parallel execution.

An example from the field of linear algebra is the second one. This is an illustration of distributed matrix multiplication algorithm. It shows that the Templet implementation of the actor model is well suited both for shared and distributed parallel architectures. The business process model example is the third one. The Templet DSL can be used to model and analyze concurrency in non-technical systems, for example, in the area of business process modeling. We studied a business scenario written in a human language and composed a formal specification for the scenario in the Templet language. The static type analysis, debugging, and testing of the program were used to verify the correctness of the specification. In particular, we compared programmatically generated event sequences with expected sequences for the studied business process. This example illustrates that in our approach much of model verification is done by $\mathrm{C}++$ compiler and the Templet runtime. 


\section{Related works}

The experimental implementation of the domain-specific language toolkit showed the following benefits of our approach.

Additional language constructions are not required to explain the meaning of an algorithm with concurrent control. This is similar to approach based on object-oriented libraries STL [7], TBB [8], CCR [9], Boost [10], and others. However, the markup and preprocessing technique reduces the amount of manual coding.

More reliable protection against programming errors is provided. This feature is compatible with modern concurrent programming languages Go [11], Occam [12], Limbo [13], Erlang [14], and some others. Static type checking in the C++ language helps to prevent incorrect connection of message source and message recipient. Semantic checking can also be implemented at the preprocessor level. For example, the Templet preprocessor can check the attainability of a state in the communication protocol for channels and the possibility to call a method for processes. The check can also be carried out during the program execution: if pair of processes does not perform a prescribed messaging protocol, calculations will stop.

Behavior of the Templet program can be investigated in more detail by means of problem-oriented debugger. The mapping algorithm can add code to provide information to the debugger. The performance prediction of a parallel program is also possible. Discrete event simulation library can easily replace standard execution mechanism for the performance analysis.

The markup language is a mean of skeleton programming and code reuse [15, 16]. One can design a universal skeleton for programs with similar control flow and adapt it to specific applications. The adaptation is made by changing $\mathrm{C}++$ code of message variables and handlers. This technique is useful for programming multi-core and many-core systems $[17,18]$.

The markup language defines concurrent execution with sequential code. A number of well-known [19,2 0] and experimental [21,22] tools for defining iterative or recursive parallelism are based on markup. We adopted the same method for an actor model of parallel execution.

The DSL language can be applied to different general-purpose programming languages. It is compatible with a number of modern industrial process control software development technologies [23, 24].

\section{Conclusion}

Our research shows a practical interest of the DSL-based approach for parallel actororiented programming. We got a fully working but relatively compact implementation of the actor language. This implementation had been deployed online as a part of the web service TempletWeb (http://template.ssau.ru/templet). 


\section{Acknowledgements}

The research was supported by the Ministry of Education and Science of the Russian Federation within the framework of the Program designed to increase the competitiveness of Samara National Research University among the world's leading scientific and educational centers over the period from 2013 till 2020. This work was partially supported by the RFBR grant 15-08-05934 A.

\section{References}

1. Vostokin S. Templet: a markup language for concurrent programming. arXiv preprint 2014; arXiv:1412.0981.

2. Ward MP. Language-oriented programming. Software-Concepts and Tools, 1994; 15(4): 147-161.

3. Dmitriev S. Language oriented programming: The next programming paradigm. JetBrains onBoard, 2004; 1(2):1-13.

4. Hoare C. Communicating sequential processes. Chapter in book: The origin of concurrent programming. Springer, 2002; 413-443.

5. Wirth N. The programming language Oberon. Software: Practice and Experience, 1988; 18(7): 671-690.

6. Hewitt C, Bishop P, Steiger R. A universal modular actor formalism for artificial intelligence. in Proc. of the 3rd international joint conference on Artificial intelligence, 1973; 235-245.

7. Stroustrup B. The $\mathrm{C}++$ programming language. Pearson Education, 2013.

8. Reinders J. Intel threading building blocks: outfitting C++ for multi-core processor parallelism. O’Reilly Media, Inc., 2007.

9. Richter J. Concurrent affairs-concurrency and coordination runtime. Louisville: MSDN Magazine, 2006; 117-128.

10. Schäling B. The boost $\mathrm{C}++$ libraries. Boris Schäling, 2011.

11. The Go programming language specification. Google Inc. 2009. Source: <http://golang.org/doc/go_spec.html>.

12. Occam programming manual. INMOS Limited. Prentice Hall Direct; 1984.

13. Ritchie DM. The Limbo programming language. Inferno Programmer(TM) Manual, 1997; 2.

14. Larson J. Erlang for concurrent programming. Communications of the ACM, 2009; 52(3): 48-56.

15. Cole M. Bringing skeletons out of the closet: a pragmatic manifesto for skeletal parallel programming. Parallel computing, 2004; 30(3): 389-406.

16. González-Vélez H, Leyton M. A survey of algorithmic skeleton frameworks: high-level structured parallel programming enablers. Software: Practice and Experience, 2010; 40(12): 1135-1160.

17. Aldinucci M, Danelutto M, Kilpatrick P. Skeletons for multi/many-core systems. in Proc. of PARCO, 2009; 265-272.

18. Karasawa Y, Iwasaki H. A parallel skeleton library for multi-core clusters. in Parallel Processing, 2009. ICPP'09. International Conference on. IEEE, 2009; 84-91.

19. Dagum L, Menon R. OpenMP: an industry standard API for shared-memory programming. Computational Science \& Engineering, IEEE, 1998; 5(1): 46-55. 
20. Blumore R, Joerg C, Kuszmaul B, Leiserson C, Randall K, Zhou Y. Cilk: An efficient multithreaded runtime system. Journal of parallel and distributed computing, 1996; 37(1): 55-69.

21. Konovalov N, Krukov V, Sazanov Y. "C-DVM - a language for the development of portable parallel programs. Programming and Computer Software, 1999; 25(1): 46-55.

22. Abramov S, Adamovich A, Inyukhin A, Moskovsky A, Roganov V, Shevchuk E, Shevchuk Y, Vodomerov A. OpenTS: an outline of dynamic parallelization approach. in Parallel Computing Technologies. Springer, 2005; 303-312.

23. Atkinson C, Kuhne T. Model-driven development: a metamodeling foundation. Software, IEEE, 2003; 20(5): 36-41.

24. Selic B. The pragmatics of model-driven development. IEEE software, 2003; 20(5): 19-25. 